\title{
Design and Baseline Characteristics of the Finerenone in Reducing Kidney Failure and Disease Progression in Diabetic Kidney Disease Trial
}

\author{
George L. Bakris $^{a}$ Rajiv Agarwal ${ }^{b}$ Stefan D. Anker ${ }^{c}$ Bertram Pitt $^{d}$ \\ Luis M. Ruilope ${ }^{\mathrm{e}-\mathrm{g}} \quad$ Christina Nowack $^{\mathrm{h}}$ Peter Kolkhof $^{\mathrm{i}}$ \\ Anna C. Ferreiraj ${ }^{j}$ Patrick Schloemerk Gerasimos Filippatos ${ }^{l, m}$ \\ on behalf of the FIDELIO-DKD study investigators
}

\begin{abstract}
${ }^{a}$ Department of Medicine, University of Chicago Medicine, Chicago, IL, USA; ${ }^{b}$ Richard L. Roudebush VA Medical Center and Indiana University, Indianapolis, IN, USA; ' Department of Cardiology and Berlin Institute of Health Center for Regenerative Therapies, German Centre for Cardiovascular Research Partner Site Berlin, Charité Universitätsmedizin, Berlin, Germany; ${ }^{d}$ Department of Medicine, University of Michigan School of Medicine, Ann Arbor, MI, USA; ${ }^{e}$ Cardiorenal Translational Laboratory and Hypertension Unit, Institute of Research i+12, Hospital Universitario 12 de Octubre, Madrid, Germany; ${ }^{f}$ CIBER-CV, Hospital Universitario 12 de Octubre, Madrid, Spain; ${ }^{9}$ Faculty of Sport Sciences, European University

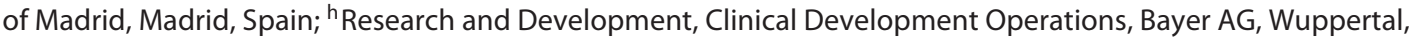
Germany; ${ }^{i}$ Research and Development, Preclinical Research Cardiovascular, Bayer AG, Wuppertal, Germany; ${ }^{j}$ Research and Development, Clinical Operations, Bayer SA, São Paulo, Brazil; k Research and Development, Statistics and Data Insights, Bayer AG, Berlin, Germany; 'Department of Cardiology, Attikon University Hospital, Athens, Greece; ${ }^{\mathrm{m}}$ University of Cyprus, Medical School, Nicosia, Cyprus
\end{abstract}

\section{Keywords}

Kidney · Mineralocorticoid · Diabetes · Aldosterone ·

Outcomes · Clinical

\begin{abstract}
Background: Among diabetics, those with kidney disease have exceptionally high rates of cardiovascular (CV) morbidity and mortality, and progression of their underlying disease. Finerenone is a novel, non-steroidal, selective mineralocorticoid-receptor antagonist which has shown to reduce albuminuria in type 2 diabetes (T2D) patients with chronic kidney disease (CKD), while revealing only a low risk of hyperkalemia. However, the effect of finerenone on renal and CV outcomes has not been investigated in long-term trials yet. Methods: The Finerenone in Reducing Kidney Failure and Disease Progression in Diabetic Kidney Disease (FIDELIO-DKD) trial aims to assess the efficacy and safety of
\end{abstract}

\begin{tabular}{ll}
\hline KARGER & $\begin{array}{l}\text { (c) } 2019 \text { The Author(s) } \\
\text { Published by S. Karger AG, Basel }\end{array}$ \\
E-Mail karger@karger.com & $\begin{array}{l}\text { This article is licensed under the Creative Commons Attribution- } \\
\text { NonCommercial-NoDerivatives 4.0 International License (CC BY- } \\
\text { NC-ND) (http://www.karger.com/Services/OpenAccessLicense). } \\
\text { Usage and distribution for commercial purposes as well as any dis- } \\
\text { tribution of modified material requires written permission. }\end{array}$
\end{tabular}

finerenone compared to placebo at reducing clinically important renal and CV outcomes in T2D patients with CKD. FIDELIO-DKD is a randomized, double-blind, placebo-controlled, parallel-group, event-driven trial running in 47 countries with an expected duration of approximately 5.5 years. FIDELIO-DKD randomized 5,734 patients with an estimated glomerular filtration rate $($ eGFR) $\geq 25-<75 \mathrm{~mL} / \mathrm{min} / 1.73$ $\mathrm{m}^{2}$ and albuminuria (urinary albumin-to-creatinine ratio $\geq 30-\leq 5,000 \mathrm{mg} / \mathrm{g}$ ). The study has at least $90 \%$ power to detect a $20 \%$ reduction in the risk of primary outcome (overall two-sided significance level $a=0.05$ ), the composite of time to first occurrence of kidney failure, a sustained decrease of eGFR $\geq 40 \%$ from baseline over at least 4 weeks, or renal death. Conclusion: FIDELIO-DKD will determine whether an

FIDELIO-DKD Phase 3 investigators and site list are included in the online supplementary files
George L. Bakris, MD

Department of Medicine, University of Chicago

5841 S. Maryland Avenue

MC 1027, Chicago, IL 60637 (USA)

E-Mail gbakris@gmail.com 
optimally treated cohort of T2D patients with CKD at high risk of renal and $C V$ events will experience cardiorenal benefits with the addition of finerenone to their treatment regimen.

(c) 2019 The Author(s)

Published by S. Karger AG, Basel

\section{Background}

Worldwide, over 2.5 million people receive renal replacement therapy, and this number is projected to grow and be more than double in 2030 [1]. Type 2 diabetes (T2D) is the most common cause of end-stage renal disease (ESRD) in the Western world, except for Taiwan, which has a higher prevalence of ESRD than the US [2]. Moreover, this trend in ESRD is rapidly increasing in Asia and Latin America [1].

The current pharmacological strategy to reduce the rate of progression of kidney disease is the optimization of blood pressure control, lipid levels, and glycemic control, and has been in place since the 1990s [3]. Following the advent of the angiotensin-receptor blocker (ARB) trials almost 20 years ago $[4,5]$, the sodium-glucose cotransporter-2 (SGLT2) inhibitor canagliflozin added to a renin - angiotensin system (RAS) blocker recently demonstrated a $34 \%$ relative risk reduction of the renal-specific composite endpoint of ESRD, a doubling of creatinine level, or death from renal causes [6]. For many years, T2D patients who have chronic kidney disease (CKD) and albuminuria $>300 \mathrm{mg}$ /day have had guideline directives to use angiotensin-converting enzyme inhibitors (ACEIs) or ARBs [7, 8]. From mid-June 2019 onwards, SGLT2 inhibitors have been recommended in addition for patients with T2D with albuminuria $>300 \mathrm{mg} / \mathrm{g}$ if their estimated glomerular filtration rate (eGFR) is $>30 \mathrm{~mL} / \mathrm{min} / 1.73 \mathrm{~m}^{2}$, to reduce cardiovascular (CV) events, with increasing evidence that they may also slow kidney disease progression [9].

While optimizing therapy with RAS blockers and SGLT2 inhibitors has markedly slowed CKD progression, it has not stopped it fully $[6,10]$. Despite the use of ACEIs, ARBs, and the concomitant use of SGLT2 inhibitors, the rate of ESRD remains unacceptably high, with more than twice the normal decline in kidney function [6]. Mineralocorticoid receptor antagonists (MRAs) such as spironolactone demonstrate renoprotective effects in preclinical studies [11, 12] and are known to reduce albuminuria and blood pressure in patients with kidney disease, well-documented surrogates of CKD progression in men [13-15]. Thus, antagonism of mineralocorticoid receptor (MR) might be effective in slowing CKD progression in T2D [16-19].
Finerenone is a novel, non-steroidal, selective MRA that has much greater MR selectivity than spironolactone and better MR affinity than eplerenone in vitro [20]. Furthermore, unlike spironolactone and eplerenone, which reach higher concentrations in renal tissue in comparison to cardiac tissue [21], finerenone is distributed relatively equally between the heart and the kidney, at least in rodents [22]. Lastly, its non-steroidal structure allows it to bind to the MR with high affinity and to inhibit recruitment of transcriptional co-activators involved in the expression of hypertrophic and pro-fibrotic genes more effectively than steroidal MRAs $[23,24]$. This unique binding mode and lack of other hormonal stimulation compared to spironolactone mitigates against untoward effects like gynecomastia $[25,26]$.

In a trial involving T2D patients with a clinical diagnosis of CKD (Mineralocorticoid-Receptor Antagonist Tolerability Study-Diabetic Nephropathy), finerenone compared to placebo showed only small mean changes in serum potassium and a low incidence of hyperkalemia leading to a low number of discontinuations with no evidence of a dose response relationship. Furthermore, finerenone compared to placebo demonstrated a statistically significant reduction in urinary albumin-to-creatinine ratio (UACR) at doses starting from $7.5 \mathrm{mg}$ on top of standard of care (including an ACEI or ARB) after 90 days of treatment [27].

These findings provided the rationale for initiating a large-scale Phase III program with finerenone investigating renal and CV outcomes in T2D patients with all stages of CKD in 2 large-scale outcome trials. In this study, we describe the design of the Finerenone in reducing Kidney Failure and Disease Progression in Diabetic Kidney Disease trial (FIDELIO-DKD; ClinicalTrials.gov identifier: NCT02540993) and present the baseline characteristics of enrolled patients.

\section{Patients and Methods}

\section{General Description}

FIDELIO-DKD is an international, multicenter, randomized, double-blind, placebo-controlled, parallel-group, event-driven study, formally designed to assess whether finerenone reduces cardiorenal morbidity and mortality in T2D patients with CKD when used in addition to standard of care.

\section{Study Population}

The inclusion and exclusion criteria are summarized in Table 1. Patients included in the trial are aged $\geq 18$ years with T2D and a clinical diagnosis of CKD, pre-treated with ACEIs or ARBs for at least 4 weeks before the run-in visit, and had serum potassium $\leq 4.8 \mathrm{mEq} / \mathrm{L}$ at both the run-in and screening visits. Patients had to 
Table 1. Inclusion and exclusion criteria

Inclusion criteria

Written informed consent signed before any study-specific procedure

Men or women aged 18 years and older

Women of child-bearing potential with a negative pregnancy test and agreeing to use adequate contraception

Patients with T2D mellitus as defined by the American Diabetes Association

Patients with a clinical diagnosis of CKD based on either of the following criteria at the run-in and screening visits:

-Persistent high albuminuria defined as UACR $\geq 30 \mathrm{mg} / \mathrm{g}(\geq 3.4 \mathrm{mg} / \mathrm{mmol})$ but $<300 \mathrm{mg} / \mathrm{g}(<33.9 \mathrm{mg} / \mathrm{mmol})$ and

eGFR $\geq 25$ but $<60 \mathrm{~mL} / \mathrm{min} / 1.73 \mathrm{~m}^{2}$ (CKD-EPI) and presence of diabetic retinopathy in the medical history

or

-Persistent very high albuminuria defined as UACR $\geq 300 \mathrm{mg} / \mathrm{g}(\geq 33.9 \mathrm{mg} / \mathrm{mmol})$ and eGFR $\geq 25 \mathrm{but}<75 \mathrm{~mL} /$ $\min / 1.73 \mathrm{~m}^{2}$ (CKD-EPI)

Prior treatment with ACEIs and ARBs

Serum potassium $\leq 4.8 \mathrm{mmol} / \mathrm{L}$ at both the run-in and screening visits

Exclusion criteria

Medical and surgical history
Known significant non-diabetic renal disease, including clinically relevant renal artery stenosis

$\mathrm{UACR}>5,000 \mathrm{mg} / \mathrm{g}(>565 \mathrm{mg} / \mathrm{mmol})$ at the run-in or screening visit

Glycosylated hemoglobin $>12 \%(>108 \mathrm{mmol} / \mathrm{mol})$ at the run-in or screening visit

Uncontrolled arterial hypertension with mean sitting SBP $\geq 170 \mathrm{~mm} \mathrm{Hg}$ or mean sitting DBP $\geq 110 \mathrm{~mm} \mathrm{Hg}$ at the run-in visit or mean sitting SBP $\geq 160 \mathrm{~mm} \mathrm{Hg}$ or mean sitting DBP $\geq 100 \mathrm{~mm} \mathrm{Hg}$ at the screening visit

$\mathrm{SBP}<90 \mathrm{~mm} \mathrm{Hg}$ at the run-in or screening visit

Patients with a clinical diagnosis of chronic heart failure with reduced ejection fraction and persistent symptoms (New York Heart Association class II-IV) at the run-in visit

Stroke, transient ischemic cerebral attack, acute coronary syndrome, or hospitalization for worsening heart failure, in the 30 days before the screening visit

Dialysis for acute renal failure in the 12 weeks before the run-in visit

Renal allograft in place or a scheduled kidney transplant in the 12 months after the run-in visit

Known hypersensitivity to the study treatment (active substance or excipients)

Addison's disease

Hepatic insufficiency classified as Child-Pugh C

Medication and drug use

Concomitant therapy with eplerenone, spironolactone, any renin inhibitor, or potassium-sparing diuretic which cannot be discontinued at least 4 weeks before the screening visit

Concomitant therapy with both ACEIs and ARBs which cannot be discontinued for the purpose of the studies

Concomitant therapy with potent cytochrome P450 isoenzyme 3A4 inhibitors or inducers (to be stopped at least 7 days before randomization)

Other

Ane patient unsuitable for the studies and would not allow participation for the full planned study period (e.g., active malignancy or other condition limiting life expectancy to $<12$ months)

Pregnant or breast-feeding or intention to become pregnant during the studies

Previous assignment to treatment during the studies

Previous (within 30 days before randomization) or concomitant participation in another clinical study (i.e., Phase I-III clinical studies) with investigational medicinal product, except for participation in the run-in and screening periods of Studies 17,530 and 16,244

Close affiliation with the investigational site: for example, a close relative of the investigator or dependent person (e.g., employee or student of the investigational site)

ACEI, angiotensin-converting enzyme inhibitor; ARB, angiotensin receptor blocker; CKD-EPI, Chronic Kidney Disease Epidemiology Collaboration; DKD, diabetic kidney disease; DBP, diastolic blood pressure; eGFR, estimated glomerular filtration rate; SBP, systolic blood pressure; UACR, urine albumin-to-creatinine ratio; T2D, type 2 diabetes. 


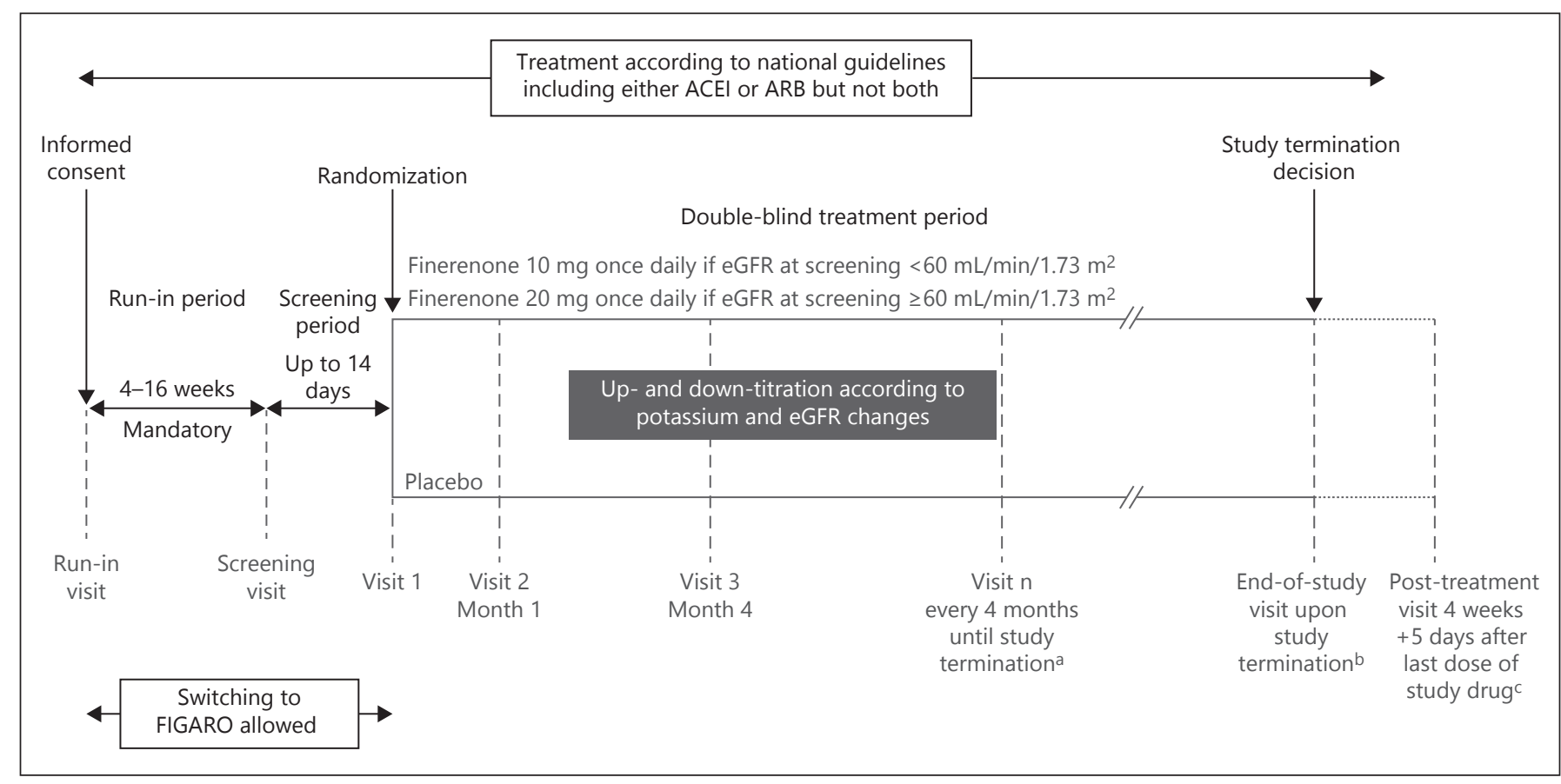

Fig. 1. Study design of FIDELIO-DKD. a Scheduled visits will continue even if the study drug treatment is discontinued; ${ }^{b}$ End-ofstudy visit will take place only after the sponsor has officially terminated the study; ${ }^{\mathrm{c}}$ For all patients who have received the study drug. ACEI, angiotensin-converting enzyme inhibitor; ARB, angiotensin-receptor blocker; eGFR, estimated glomerular filtration rate; FIGARO, Finerenone in Reducing Cardiovascular Mortality and Morbidity in Diabetic Kidney Disease. have persistent high albuminuria (defined as UACR $\geq 30$ but $<300$ $\mathrm{mg} / \mathrm{g}$ ), eGFR $\geq 25$ but $<60 \mathrm{~mL} / \mathrm{min} / 1.73 \mathrm{~m}^{2}$, and presence of diabetic retinopathy in the medical history, or persistent very high albuminuria (defined as UACR $\geq 300 \mathrm{mg} / \mathrm{g}$ ) and eGFR $\geq 25$ but $<75$ $\mathrm{mL} / \mathrm{min} / 1.73 \mathrm{~m}^{2}$.

Two recruitment caps were pre-specified and closed per region. The first cap limited the randomization of patients with high albuminuria and diabetic retinopathy in the medical history to approximately $10 \%$ of all patients randomized. The second cap limited the randomization of patients with very high albuminuria and eGFR $60-75 \mathrm{~mL} / \mathrm{min} / 1.73 \mathrm{~m}^{2}$ to $10 \%$ of all patients randomized with very high albuminuria at screening.

\section{Study Design}

The study design of FIDELIO-DKD is illustrated in Figure 1. After signing the informed consent, a run-in visit was performed. If the patient met all inclusion criteria and none of the exclusion criteria, a mandatory run-in period that could last between a minimum of 4 and a maximum of 16 weeks started. At the end of the run-in period, a screening visit to confirm the patient's eligibility again took place, with subsequent randomization within 2 weeks.

To account for the high variability of UACR and eGFR, patients were permitted to switch either after the run-in or screening visit between FIDELIO-DKD and the second study of the program, Finerenone in Reducing Cardiovascular Mortality and Morbidity in Diabetic Kidney Disease (FIGARO-DKD; ClinicalTrials.gov identifier: NCT02545049), before being randomized. As CKD in T2D is a disease continuum and variations in eGFR and UACR within the boundaries of this disease continuum do not affect the clinical risk, the switching of patients before randomization was deemed justified and necessary to reduce the burden on candidate study participants (run-in and screening assessments were not repeated after switching). A switchover from either trial was permitted only once.

Due to the anticipated recruitment period of $>2$ years and the progressive nature of the underlying disease, re-screening was permitted once. Conditions for re-screening were amended in 2017 to shorten the re-screening period from 6 to 3 months and to allow re-screening even if the reason for the initial screen failure was an elevated blood potassium value (serum potassium $>4.8 \mathrm{mEq} / \mathrm{L}$ ).

Eligible patients were randomized 1:1 via an interactive telephone/web-based system to receive once-daily treatment with either finerenone or placebo. Treatment assignment was determined by computer-generated random sequence with stratification by region (North America, Latin America, Europe, Asia, Other), eGFR category at screening $\left(25-<45,45-<60\right.$, and $\left.\geq 60 \mathrm{~mL} / \mathrm{min} / 1.73 \mathrm{~m}^{2}\right)$, and category of albuminuria at screening (high or very high).

The starting dose of study drug was selected based on eGFR measured at the screening visit: patients started on finerenone $10 \mathrm{mg} /$ day or matching placebo if their eGFR was $25-<60 \mathrm{~mL} / \mathrm{min} / 1.73 \mathrm{~m}^{2}$; if their eGFR was $\geq 60 \mathrm{~mL} / \mathrm{min} / 1.73 \mathrm{~m}^{2}$, the starting dose was $20 \mathrm{mg}$ / day. Up-titration of study drug to $20 \mathrm{mg} /$ day is permitted at any time after Visit 2 (Month 1), down-titration to $10 \mathrm{mg}$ /day at any time after start of treatment. Patients are instructed to take the study drug tablet orally, once daily at approximately the same time every day.

Central laboratory values, including serum potassium and serum creatinine, were obtained at all study visits and used for con- 
firmation of patients' eligibility, safety monitoring, renal endpoint reporting, and end-of-study statistical analysis. From Visit 1 onwards, blood potassium and serum creatinine are measured at all on-treatment visits at a local laboratory. The investigators are instructed to use these local values for dose titrations and safety monitoring. Patients' safety is monitored with physical examinations (including measurements of weight and vital signs) and 12-lead electrocardiograms at regular intervals throughout the study. $\mathrm{Pa}$ tients are assessed for adverse events and study endpoints at each visit and are requested to complete health-related quality-of-life questionnaires (EQ-5D-5L and Kidney Disease Quality of Life) at baseline and yearly thereafter.

The study protocol was approved by International Review Boards, independent Ethics Committees, and competent authorities according to national and international regulations. FIDELIODKD is conducted in accordance with the ICH Harmonised Tripartite Guideline for Good Clinical Practice. All study participants provided written informed consent before entering the study.

\section{Objectives and Endpoints}

The primary endpoint of FIDELIO-DKD is a composite of time to first occurrence of kidney failure, a sustained decrease of eGFR $\geq 40 \%$ from baseline over at least 4 weeks, or renal death. The component kidney failure is defined as either the occurrence of ESRD or eGFR $<15 \mathrm{~mL} / \mathrm{min} / 1.73 \mathrm{~m}^{2}$, confirmed by a second measurement at the earliest 4 weeks after the initial measurement. ESRD is defined as the initiation of chronic dialysis (hemo- or peritoneal dialysis) for at least 90 days or renal transplantation. The eGFR threshold of $15 \mathrm{~mL} / \mathrm{min} / 1.73 \mathrm{~m}^{2}$ is consistent with the definition of kidney failure from Kidney Disease: Improving Global Outcomes [28] and was chosen to include an objective component to the endpoint because the decision to initiate dialysis therapy or kidney transplantation may be affected by factors other than eGFR.

The main pre-specified secondary endpoint is a composite of time to first occurrence of CV mortality and morbidity, as measured by the composite endpoint of time to first occurrence of CV death, non-fatal myocardial infarction, non-fatal stroke, or hospitalization for heart failure.

Other secondary endpoints include time to all-cause mortality, time to all-cause hospitalization, change in UACR from baseline to 4 months, and a composite endpoint of time to first occurrence of kidney failure or sustained decrease in eGFR $\geq 57 \%$ from baseline over at least 4 weeks or renal death.

\section{Statistical Considerations}

Statistical Analyses

The primary efficacy endpoint for the statistical analysis is the time to first occurrence of the composite of onset of (i) kidney failure, (ii) a sustained decrease of eGFR $\geq 40 \%$ from baseline over at least 4 weeks, or (iii) renal death. Events included in the primary analysis will be counted from the day of randomization onwards until the planned end-of-study visit following the study sponsor's decision to terminate the study. Patients without a primary efficacy endpoint event will be censored at the date of their last contact with complete information on all components of the primary composite endpoint (up to and including the end-of-study visit). In case a non-renal death occurs within 5 months from the last visit (4 monthly visits plus maximum allowed time window of 30 days) and a subsequent clinic visit had been planned, the non-renal death date will be used as the censoring date.

Design and Baseline Characteristics of the FIDELIO Trial
To test for superiority of finerenone over placebo in prolonging the time to the first primary efficacy endpoint event, the null hypothesis of equal hazards in the 2 treatment groups will be evaluated via a log-rank test, stratified by the stratification factors region, eGFR category at screening, and category of albuminuria at screening. The treatment effect will be expressed as a hazard ratio with a corresponding confidence interval from a stratified Cox regression model. The statistical analyses will follow the intentionto-treat principle and will be based primarily on the full analysis set, consisting of all randomized patients considered valid for analysis. An analysis on the per protocol set, excluding patients with relevant deviations from the protocol, will be conducted as a sensitivity analysis in which only events occurring within 30 days after permanent treatment discontinuation will be considered.

To control the multiplicity arising from multiple tests, a weighted Bonferroni-Holm procedure will be used for the primary renal efficacy endpoint and the key secondary CV endpoint, followed by a hierarchical testing procedure of further secondary efficacy endpoints (as in the order given above). The weights assigned to the primary efficacy endpoint and the key secondary endpoint are given as $2 / 3$ and $1 / 3$, resulting in a minimal local two-sided alpha level of 3.3333 and $1.6667 \%$, respectively, which will be increased to $5 \%$ for one test if the null hypothesis for the other test can be rejected. The full alpha level of $5 \%$ will be passed along hierarchically to the further secondary endpoints in a serial gatekeeping approach, that is, if and only if both the primary endpoint and the key secondary endpoint show significance. Within the hierarchy of the secondary endpoints, the full alpha is forwarded to the next null hypothesis as long as the previous null hypothesis is rejected, otherwise confirmatory testing stops at this point. As described under Powering and Sample Size, the actual significance levels used during the final analysis will be slightly adjusted based on the formal interim analysis. The testing strategy is further illustrated in Figure 2. To declare the study successful, it will be considered sufficient to reject the null hypothesis related to the primary renal endpoint or key secondary CV endpoint.

\section{Powering and Sample Size}

As an event-driven study, FIDELIO-DKD has a power of $90 \%$ to detect a $20 \%$ relative risk reduction of finerenone versus placebo in the primary efficacy endpoint (equaling a hazard ratio of 0.8 ) based on a total of 1,068 patients with primary renal events and applying the log-rank test at a two-sided significance alpha level of $3.3333 \%$. Further assumptions at the planning stage included an annual placebo event rate of $12 \%$ (assumed to be unaffected by treatment discontinuations), a common annual lost-to-follow-up rate of $0.7 \%$ in both treatment groups, an annual finerenone discontinuation rate of $5 \%$, and a total treatment duration between 44 and 48 months, consisting of a recruitment period of 33 and 41 months with an equal recruitment pattern during the accrual period and a maximum treatment period of the last recruited patient of 11 and 7 months, respectively. Taking the ramp-up time during recruitment into consideration, this leads to an estimated required number of approximately 4,800 patients to be randomized. Assuming a screening failure rate of $50 \%, 9,600$ patients need to be screened. To account for the lower-than-assumed event rates for the primary endpoint as observed during the conduct of the trial, the originally planned number of randomized patients was increased by approximately 1,000 patients.

One formal interim analysis is planned to be conducted when approximately 712 primary efficacy endpoint events (i.e., $2 / 3$ of the 


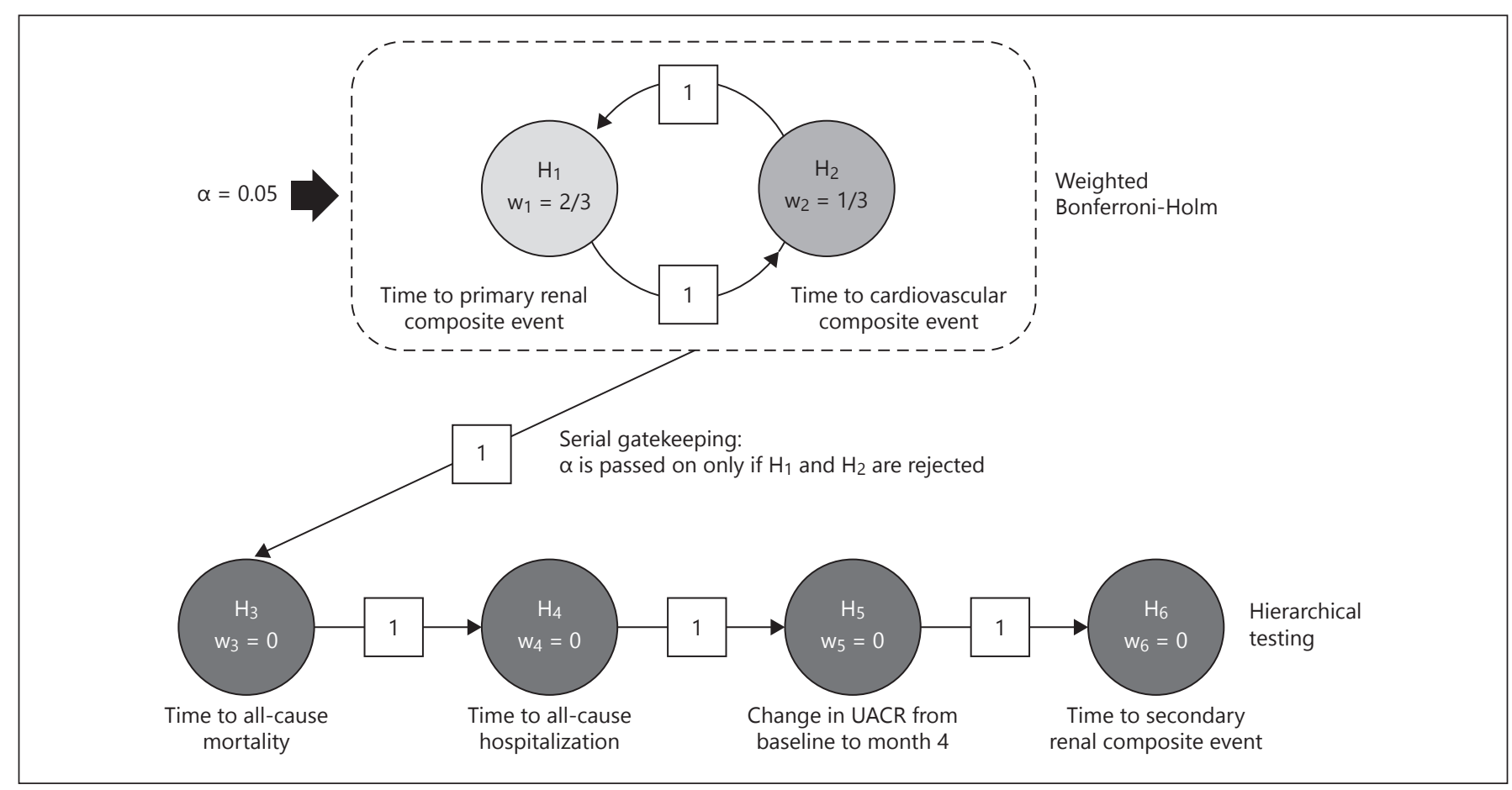

Fig. 2. Statistical testing strategy of primary and secondary efficacy endpoints for FIDELIO-DKD. UACR, urinary albumin-to-creatinine ratio.

finally intended number) have been observed. To guide the decision regarding early stopping of the study for success at the interim analysis, the Haybittle-Peto rule will be used, requiring a two-sided $p$ value below 0.00270 for both the null hypotheses corresponding to the primary renal efficacy endpoint and the key secondary $\mathrm{CV}$ endpoint to be rejected and leading to a minimal alpha adjustment for the respective tests at the final analysis stage. Additionally, for lack of efficacy, a non-binding futility approach will be used to allow stopping the study at the interim analysis stage if the conditional power of rejecting the null hypothesis related to the primary endpoint at the final stage given the interim data falls to an unacceptably low level of $10 \%$ or less.

Additional Statistical Considerations

For analysis, Austria, Belgium, Denmark, Finland, France, Germany, Ireland, Italy, Netherlands, Norway, Portugal, Spain, Sweden, Switzerland, and the United Kingdom are considered in Western Europe; Bulgaria, Czech Republic, Greece, Hungary, Lithuania, Poland, Romania, Russia, Slovakia, Turkey, and Ukraine in Eastern Europe.

\section{Results}

Recruitment of patients for FIDELIO-DKD began in September 2015 and was completed in June 2018. A total of 13,911 individuals, including 604 patients who were rescreened, were enrolled at 1,062 sites in 47 countries, re- sulting in 5,734 randomized patients. During the run-in or screening phase but before randomization, 1,374 patients switched over from FIGARO-DKD. The rate of screening failure in all patients enrolled was 58.8 and $14.9 \%$ in patients who switched. Sixty patients in FIDELIO-DKD were excluded from the full analysis set due to substantial Good Clinical Practice violations, resulting in 5,674 patients (99.0\%) in whom the primary analysis will be performed.

More than $40 \%$ of the patients were recruited in Europe, with a similar number in Western and Eastern Europe, $16.6 \%$ in North America, $10.5 \%$ in Latin America, 27.8\% in Asia, and 3.5\% in Australia, New Zealand, and South Africa (region "Other").

The overall trial population is predominately male $(70.2 \%)$ and white $(65.9 \%)$, with a mean age of 65.6 years and a mean duration of diabetes of 16.6 years. At baseline, mean glycosylated hemoglobin was $7.7 \%$, mean eGFR $44.3 \mathrm{~mL} / \mathrm{min} / 1.73 \mathrm{~m}^{2}$, mean serum potassium $4.4 \mathrm{mEq} / \mathrm{L}$, and median UACR $851 \mathrm{mg} / \mathrm{g}$. Most patients (88.4\%) had eGFR $<60 \mathrm{~mL} / \mathrm{min} / 1.73 \mathrm{~m}^{2}$ and $54.9 \%$ eGFR $<45 \mathrm{~mL} /$ $\min / 1.73 \mathrm{~m}^{2}$; the majority of patients $(87.4 \%)$ had very high albuminuria at baseline.

At baseline, 3,727 patients (65.7\%) were taking ARBs and 1,942 (34.2\%) ACEIs, as requested by the protocol, and almost all patients (97.4\%) were on glucose-lowering 
Table 2. Baseline characteristics

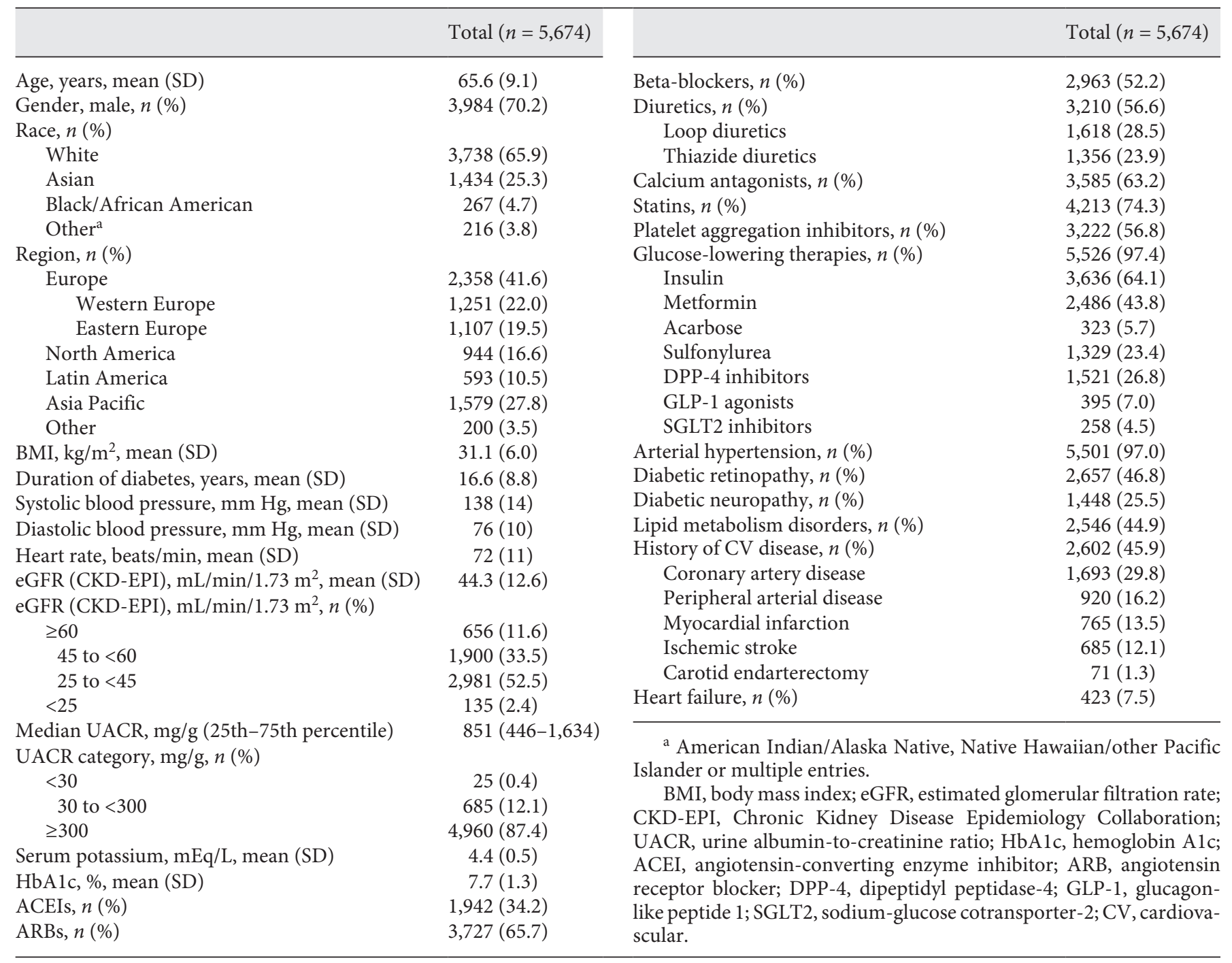

medication. Approximately 2/3 (64.1\%) were using insulin, while metformin was the most frequently used glucose-lowering oral drug at baseline. Glucagon-like peptide 1 agonists were used by $7.0 \%$ of patients, while $4.5 \%$ were using SGLT2 inhibitors.

At baseline, nearly all patients (97.0\%) had arterial hypertension as concomitant disease, and $46.8 \%$ had diabetic retinopathy. Less than half (45.9\%) had CV disease (CVD) in the medical history: $29.8 \%$ had coronary artery disease, $13.5 \%$ myocardial infarction, $12.1 \%$ ischemic stroke, $16.2 \%$ peripheral artery disease, and $1.3 \%$ had a carotid endarterectomy. Only $7.5 \%$ of all patients suffered from heart failure at baseline, which is related to the protocol-requested exclusion of all patients with heart failure with reduced ejection fraction with New York Heart Association class II-IV at run-in and screening. Further information about the baseline characteristics can be found in Table 2 and in online supplementary Table S1 (see www.karger.com/doi/10.1159/000503713).

\section{Discussion}

Treatment of CKD in general has evolved over the last few decades, resulting in a slowing of progression of kidney disease from 10 to $12 \mathrm{~mL} / \mathrm{min} /$ year to approximately $2 \mathrm{~mL} / \mathrm{min} /$ year in patients with an eGFR $<60 \mathrm{~mL} / \mathrm{min} / 1.73$ $\mathrm{m}^{2}$, normal decline being around $0.7-0.9 \mathrm{~mL} / \mathrm{min} /$ year 
[10]. Newer treatments to help glycemic control have also translated into further slowing of CKD progression (eGFR decline of $1.85 \mathrm{~mL} / \mathrm{min} /$ year) as well as further reduction of CV events in T2D patients $[6,29]$. However, despite this promising progress T2D patients with CKD still exhibit residual cardiorenal morbidity and mortality.

Since 2001, numerous trials assessing various agents with a variety of mechanisms have failed to slow CKD progression over RAS blockers. Attempts were made to reduce the high CV and renal risk in patients already taking RAS inhibitor monotherapy by adding a second RAS blocker: in the Veterans Affairs Nephropathy in Diabetes trial, an ACEI in combination with an ARB [30], and in the Aliskiren Trial in T2D using Cardiorenal Endpoints, the combination of an ARB with the renin inhibitor aliskiren were investigated [31]. Dual RAS blockade increased the frequency of serious adverse events such as acute kidney injury, hyperkalemia, or stroke, and therefore cannot be recommended in T2D patients with CKD.

More recently, the Canagliflozin and Renal Events in Diabetes with Established Nephropathy Clinical Evaluation study investigating canagliflozin in patients with T2D and CKD with very high albuminuria found a $30 \%$ risk reduction for progression to ESRD, doubling of serum creatinine, or CV or renal death over placebo in patients already receiving RAS blockers [6]. In a meta-analysis by Zelniker et al. [32], the effect of SGLT2 inhibitors on major adverse CV events (i.e., myocardial infarction, stroke, or CV death) and renal outcomes was analyzed. SGLT2 inhibitors reduced major adverse CV events by $11 \%$, with benefit seen only in patients with atherosclerotic CVD (14\% risk reduction) and not in those without. SGLT2 inhibitors reduced the risk of CV death or hospitalization for heart failure by $23 \%$, with a similar benefit in patients with and without atherosclerotic CVD and with and without a history of heart failure. The magnitude of benefit of SGLT2 inhibitors varied with baseline renal function and showed a lesser reduction in progression of renal disease in patients with more severe kidney disease at baseline.

The use of SGLT2 inhibitors was very low in general when FIDELIO-DKD started recruitment in September 2015. Despite not restricting the use of SGLT2 inhibitors in enrolled patients, it was even lower in the targeted study population, since the initiation of treatment with SGLT2 inhibitors in patients with eGFR $<60 \mathrm{~mL} /$ $\mathrm{min} / 1.73 \mathrm{~m}^{2}$ was prohibited, and it was recommended to stop treatment with SGLT2 inhibitors in patients with eGFR persistently $<45 \mathrm{~mL} / \mathrm{min} / 1.73 \mathrm{~m}^{2}$. At baseline, $4.5 \%$ of all randomized patients were treated with SGLT2 in- hibitors. During the trial, the use of SGLT2 increased slightly but is still low ( $<10 \%$ of patients). Most likely the data collected will not be sufficient for a meaningful subgroup analysis. However, in previous trials treatment with an MRA has never been investigated on top of a RAS blocker in this group of non-heart failure patients and hence the results of FIDELIO-DKD will be able to generate hypotheses for further investigations.

Inflammation and fibrosis mediated by MR overactivation are also observed in the kidney [33]. The resulting glomerular podocyte injury and mesangial cell proliferation may manifest as glomerular injury, renal vasoconstriction, and albuminuria. Targeting MR overactivation with MRAs, based on the studies conducted so far, showed a sustained reduction in albuminuria of $\geq 30 \%$, a biomarker that has now been validated as surrogate for slowing CKD progression $[13,34,35]$. It is therefore reasonable to assume that MR blockade, if safe, may offer additional benefit when added to monotherapy with a RAS blocker by attenuating aldosterone breakthrough, and through inhibition of deleterious effects of aldosterone, such as renal inflammation and fibrosis.

The currently available steroidal MRAs, spironolactone and eplerenone, have been investigated in clinical studies that varied between 4 and 52 weeks in duration and assessed the effects on albuminuria in different CKD populations. However, to date, all studies are plagued with hyperkalemia as a major complication of this class. In a recent meta-analysis, Yang et al. [36] concluded that treatment with spironolactone in patients with moderate to severe (stage 3-4) CKD was associated with a lower risk of ESRD (relative risk 34\%) but was complicated by a higher risk of hyperkalemia-associated hospitalization (3 times greater risk). They concluded that the renoprotective benefit of spironolactone may be offset by the hyperkalemia risk. In another clinical study of heart failure patients, Trevisan et al. [37] concluded that in an unselected cohort of new users of MRAs, hyperkalemia was very common and frequently followed by therapy interruption, especially among participants with CKD. As a result, the median time on MRA treatment during the initial 12 months of treatment was 179 days (130346 days), with $24 \%$ of remaining patients on therapy after 12 months. Participants with CKD were at highest risk of hyperkalemia and discontinuation. In an investigation analyzing electronic medical records of a diverse US population, Collins et al. [38] found that serum potassium elevation even above $5 \mathrm{mEq} / \mathrm{L}$ was associated with an increase in $\mathrm{CV}$ risk. Hyperkalemia requiring dose reduction or even discontinuation of a RAS inhibitor may also have 
a negative impact on the prognosis in $\mathrm{T} 2 \mathrm{D}$ patients with CKD or heart failure [38].

Hyperkalemia is a disabler of renal function-preserving medications. In a post hoc analysis from the Reduction of Endpoints in NIDDM with the Angiotensin II Antagonist Losartan study, Miao et al. [39] demonstrated that patients who achieved mean serum potassium $\geq 5 \mathrm{mEq} / \mathrm{L}$ during follow-up had a $43 \%$ higher risk of the composite endpoint of doubling of serum creatinine or ESRD after controlling for potential confounders. They concluded that treatment with losartan is associated with a high risk of elevated serum potassium levels in patients with diabetes mellitus and nephropathy. These elevated serum potassium levels are in turn associated with an increased risk of renal progression and offset the renoprotective effects of losartan. Given that the major risk factors for hyperkalemia are eGFR $<45 \mathrm{~mL} / \mathrm{min} / 1.73 \mathrm{~m}^{2}$ or serum potassium $>4.5 \mathrm{mEq} / \mathrm{L}$ on diuretics [40], it is clear that those with CKD in T2D patients will be at highest risk, so we restricted recruitment to patients with potassium levels up to $4.8 \mathrm{mEq} / \mathrm{L}$.

Finerenone is a novel, non-steroidal, selective MRA that is associated with lower rates of hyperkalemia compared to steroidal MRAs [41]. In the MineralocorticoidReceptor Antagonist Tolerability Study-Diabetic Nephropathy Study, finerenone induced a dose-dependent reduction of UACR, with the largest placebo-corrected reduction $(-38 \%)$ in the group being treated with $20 \mathrm{mg}$ once daily. Finerenone dosages over 90 days of treatment resulted in small declines in eGFR $(\sim 2-4 \mathrm{~mL} / \mathrm{min} / 1.73$ $\left.\mathrm{m}^{2}\right)$ and blood pressure $(3-5 \mathrm{~mm} \mathrm{Hg})$ as well as small changes in serum potassium $(+0.2 \mathrm{mEq} / \mathrm{L})$ [27].

FIDELIO-DKD is the largest renal study recruited to specifically investigate the progression of kidney disease in T2D patients with CKD. While the primary focus is on renal outcomes, potential benefits on $\mathrm{CV}$ events will be assessed as well. Unlike previous outcome trials where the levels of albuminuria and kidney function were advanced into stages 3 and 4, FIDELIO-DKD has also included a cohort of patients with lower levels of albuminuria (high albuminuria defined as UACR $\geq 30$ but $<300 \mathrm{mg} / \mathrm{g}$ ) and stage 2 CKD (eGFR $60-75 \mathrm{~mL} / \mathrm{min} / 1.73 \mathrm{~m}^{2}$ ). FIDELIO-DKD is part of a larger Phase III program that consists of 2 largescale, event-driven outcome trials, FIDELIO-DKD and FIGARO-DKD, an event-driven trial investigating the cardiorenal effects of finerenone in T2D patients with less advanced kidney disease. Complemented by the results of FIGARO-DKD where even T2D patients with stage $2 \mathrm{CKD}$ with high and very high albuminuria and stage $1 \mathrm{CKD}$ patients with very high albuminuria are included, the pro- gram will allow evaluation of the effects of finerenone in the broadest spectrum of CKD patients with T2D ever investigated. Finerenone was developed neither as an antihypertensive agent nor as a glucose-lowering drug being repurposed for a population with kidney impairment. Instead, it was designed to alleviate disease burden in patients with cardiorenal diseases, especially in T2D patients.

The results of FIDELIO-DKD will determine whether an optimally treated cohort of T2D patients with CKD at high risk of progression of their renal disease and CV events will experience cardiorenal benefits with the addition of finerenone to their treatment regimen. Results are expected in 2020 .

\section{Acknowledgements}

The authors express their sincere appreciation to all involved colleagues in the study centers for their participation in this trial. The authors would like to acknowledge all the patients who are participating in this trial. The authors thank the study coordinators Stefania Collamati (Bayer SpA, Milan, Italy), Michelle King, Laurence West, Angela May (all Bayer PLC, Reading, UK), Anna Lindroth (Bayer AB, Solna, Sweden), Cecilia Fedorov (PRA Health Sciences, Bayer Inc., Mississauga, Canada), Lydia Christopher (SLS Services, Bayer PLC, Reading, UK), GoUne You (Bayer Korea Ltd., Seoul, South Korea), Dirk Alta (Bayer B.V., Mijdrecht, The Netherlands), data managers Jeannette Gerstner, Jacobus Buytendach (Bayer AG, Berlin, Germany), and Martin Gregory (PRA Health Sciences, Bayer AG, Berlin, Germany), and Statistical Analysts Cosima Klein, Aziz Tuemer and Dominik Habel (all Bayer AG, Berlin, Germany). Special thanks to Meike Brinker (Bayer AG, Wuppertal, Germany) for her continuous support of the program in different important roles and to UI Yen Morgenthaler (Bayer AG, Berlin, Germany) for her continuous Medical Writing support.

\section{Statement of Ethics}

The study protocol was approved by International Review Boards, independent Ethics Committees, and competent authorities according to national and international regulations. FIDELIODKD is conducted in accordance with the ICH Harmonised Tripartite Guideline for Good Clinical Practice. All participants provided written, informed consent before entering the study.

\section{Disclosure Statement}

G.L.B. reports research funding, paid to the University of Chicago Medicine, from Bayer, Janssen, and Vascular Dynamics; he acted as a consultant for Merck, Vascular Dynamics, Relypsa, Boehringer Ingelheim, Sanofi, Pfizer, Novo Nordisk, Ionis, and AstraZeneca; is an editor of American Journal of Nephrology, Nephrology, and Hypertension, and section editor of UpToDate; and is an associate editor of Diabetes Care and Hypertension Research. 
R.A. is a member of data safety monitoring committees for AstraZeneca and Ironwood Pharmaceuticals; a member of steering committees of randomized trials for Akebia, Bayer, Janssen, GlaxoSmithKline, Relypsa, and Sanofi and Genzyme US Companies; a member of adjudication committees for Bayer, Boehringer Ingelheim, and Janssen; and a member of a scientific advisory board or a consultant for Bird Rock Bio, Celgene, Daiichi Sankyo, Inc., Eli Lilly, Relypsa, Reata, Takeda Pharmaceuticals, USA, and ZS Pharma; he has served as associate editor of the American Journal of Nephrology and Nephrology Dialysis and Transplantation and has been an author for UpToDate; and he has received research grants from the U.S. Veterans Administration and the National Institutes of Health. S.D.A. has received research support from Abbott Vascular and Vifor International, and personal fees from Boehringer Ingelheim, Bayer, AstraZeneca, Novartis, Vifor International, Impulse Dynamics, Respicardia, and St. Jude Medical. B.P. reports consultant fees for Bayer, AstraZeneca, Sanofi, scPharmaceuticals, SQ Innovation, G3 Pharmaceuticals, Sarfez, Vifor, Cereno, Ardelyx, KBP Biosciences and Windtree Pharmaceuticals; he has stock options for KBP Biosciences, SQ Innovation, Sarfez, scPharmaceuticals, Cereno and G3 Pharmaceuticals, and Relypsa; he also holds a patent for site-specific delivery of eplerenone to the myocardium (US patent \#9931412). L.M.R. has served as advisor/ speaker for AstraZeneca, Bayer, Daiichi Sankyo, Medtronic, Novartis, and Recordati. C.N., P.K., and P.S. are full-time employees of Bayer AG, Division Pharmaceuticals, Germany. A.C.F. is a fulltime employee of Bayer S.A., Brazil. G.F. reported that he is a committee member of trials and registries sponsored by Bayer, Novartis, Servier, Vifor, Medtronic, and Boehringer Ingelheim.

\section{Funding Sources}

FIDELIO-DKD is a research study conducted and funded by Bayer AG.

\section{Author Contributions}

The sponsor designed the study in conjunction with the Executive Committee (EC). G.L.B. wrote the first draft of the paper, had full access to the study design information, and had final responsibility for the decision to submit for publication. All authors provided input for the preparation of subsequent drafts and approved the final version for submission. Technical editorial assistance was provided by Lynda Chang, $\mathrm{PhD}$, of McCann Health, and was funded by Bayer AG. All authors reviewed and approved the manuscript.

\section{Appendix}

\section{Program Management and Committees}

FIDELIO-DKD is a research study conducted and funded by Bayer AG. The study protocol was written by the sponsor in close collaboration with the members of the EC.

The EC supervised the conduct of the program, supported the protocol development, and provided ongoing scientific guidance. The members of the EC are G.L.B., G.F., B.P., and L.M.R., as well as R.A. (Chairman of the Renal Clinical Event Committee) and S.D.A. (Chairman of the Cardiovascular Clinical Event Committee). A steering committee including the EC members, worldwide country lead investigators, and additional content experts is responsible for scientific guidance and local implementation of the protocols. The authors are responsible for drafting and editing this design and baseline paper and its content.

\section{Data Monitoring Committee}

Periodic assessments of safety and efficacy are performed in FIDELIO-DKD by an independent Data Monitoring Committee, which also oversees the safety and efficacy in FIGARO-DKD. One interim analysis for overwhelming efficacy in terms of reducing the primary renal efficacy endpoint as well as the main secondary $\mathrm{CV}$ endpoint was pre-specified to occur after accrual of $67 \%$ of the planned primary renal efficacy endpoints. Members of the independent Data Monitoring Committee are Murray Epstein (Chair), Aldo P. Maggioni (Chair), Glenn M. Chertow, Gerald DiBona, Jean Rouleau, Jose Lopez-Sendon, and Tim Friede (Statistician).

\section{Central Clinical Event Committee}

All potential outcome events are independently reviewed and adjudicated by a Clinical Event Committee, which consists of 3 sub-committees (cardiac, renal, and neuro). All potential outcome events are randomly distributed to 2 members of the appropriate sub-committee. If there is disagreement between the 2 initial reviewers, the event will be distributed to a third reviewer. If the third reviewer does not agree with either of the first 2 reviewers, the event will be reviewed at a panel meeting to reach a decision.

Members of the Renal Clinical Event Committee are R.A. (Chair), Pantelis Sarafidis, Alan Jardine, Phyllis August, Sankar Navaneethan, and Titte Srinivas; members of the Cardiac Clinical Event Committee are S.D.A. (Chair), Andrew Coats, Piotr Ponikowski, John Teerlink, Barry Greenberg, James Januzzi, Stephan von Haehling, and Wolfram Doehner; members of the Neuro Clinical Event Committee are Wolfram Doehner (Chair), Turgut Tatlisumak, Lauren Phillips, Carlos Kase, and Hans Diener.

\section{References}

1 Liyanage T, Ninomiya T, Jha V, Neal B, Patrice HM, Okpechi I, et al. Worldwide access to treatment for end-stage kidney disease: a systematic review. Lancet. 2015 May;385(9981): 1975-82.

2 Wu PP, Kor CT, Hsieh MC, Hsieh YP. Association between end-stage renal disease and incident diabetes mellitus-A nationwide population-based cohort study. J Clin Med. 2018 Oct;7(10):E343.

3 Gaede $\mathrm{P}$, Lund-Andersen $\mathrm{H}$, Parving $\mathrm{HH}$, Pedersen O. Effect of a multifactorial intervention on mortality in type 2 diabetes. $\mathrm{N}$ Engl J Med. 2008 Feb;358(6):580-91.
4 Brenner BM, Cooper ME, de Zeeuw D, Keane WF, Mitch WE, Parving $\mathrm{HH}$, et al.; RENAAL Study Investigators. Effects of losartan on renal and cardiovascular outcomes in patients with type 2 diabetes and nephropathy. N Engl J Med. 2001 Sep; 345(12):861-9. 
5 Lewis EJ, Hunsicker LG, Clarke WR, Berl T, Pohl MA, Lewis JB, et al.; Collaborative Study Group. Renoprotective effect of the angiotensin-receptor antagonist irbesartan in patients with nephropathy due to type 2 diabetes. N Engl J Med. 2001 Sep;345(12): 851-60.

6 Perkovic V, Jardine MJ, Neal B, Bompoint S, Heerspink HJ, Charytan DM, et al.; CREDENCE Trial Investigators. Canagliflozin and renal outcomes in type 2 diabetes and nephropathy. N Engl J Med. 2019 Jun;380(24): 2295-306.

7 American Diabetes Association. Standards of medical care in diabetes. 2019. [accessed July 10, 2019]. Available from: https://care.diabetesjournals.org/content/diacare/suppl/2018/12/17/42.Supplement_1.DC1/ DC_42_S1_2019_UPDATED.pdf.

8 Whelton PK, Carey RM, Aronow WS, Casey DE Jr, Collins KJ, Dennison Himmelfarb C, et al. 2017 ACC/AHA/AAPA/ABC/ACPM/ AGS/APhA/ASH/ASPC/NMA/PCNA guideline for the prevention, detection, evaluation, and management of high blood pressure in adults: a report of the American College of Cardiology/American Heart Association Task Force on Clinical Practice Guidelines. Hypertension. 2018 Jun;71(6):e13-115.

9 American Diabetes Association. Living standards of medical care in diabetes. 2019. [accessed July 30, 2019]. Available from: https:// care.diabetesjournals.org/living-standards\# June.

10 Sternlicht H, Bakris GL. The kidney in hypertension. Med Clin North Am. 2017 Jan; 101(1):207-17.

11 Blasi ER, Rocha R, Rudolph AE, Blomme EA, Polly ML, McMahon EG. Aldosterone/salt induces renal inflammation and fibrosis in hypertensive rats. Kidney Int. 2003 May;63(5): 1791-800.

12 Guo C, Martinez-Vasquez D, Mendez GP, Toniolo MF, Yao TM, Oestreicher EM, et al. Mineralocorticoid receptor antagonist reduces renal injury in rodent models of types 1 and 2 diabetes mellitus. Endocrinology. 2006 Nov; 147(11):5363-73.

13 Bomback AS, Kshirsagar AV, Amamoo MA, Klemmer PJ. Change in proteinuria after adding aldosterone blockers to ACE inhibitors or angiotensin receptor blockers in CKD: a systematic review. Am J Kidney Dis. 2008 Feb; 51(2):199-211.

14 Ando K, Ohtsu H, Uchida S, Kaname S, Arakawa Y, Fujita T; EVALUATE Study Group. Anti-albuminuric effect of the aldosterone blocker eplerenone in non-diabetic hypertensive patients with albuminuria: a double-blind, randomised, placebo-controlled trial. Lancet Diabetes Endocrinol. 2014 Dec;2(12):944-53.

15 Shavit L, Lifschitz MD, Epstein M. Aldosterone blockade and the mineralocorticoid receptor in the management of chronic kidney disease: current concepts and emerging treatment paradigms. Kidney Int. 2012 May; 81(10):955-68.
16 Epstein M, Williams GH, Weinberger M, Lewin A, Krause S, Mukherjee R, et al. Selective aldosterone blockade with eplerenone reduces albuminuria in patients with type 2 diabetes. Clin J Am Soc Nephrol. 2006 Sep;1(5): 940-51.

17 Mehdi UF, Adams-Huet B, Raskin P, Vega $\mathrm{GL}$, Toto RD. Addition of angiotensin receptor blockade or mineralocorticoid antagonism to maximal angiotensin-converting enzyme inhibition in diabetic nephropathy. J Am Soc Nephrol. 2009 Dec;20(12):264150.

18 Rossing K, Schjoedt KJ, Smidt UM, Boomsma F, Parving HH. Beneficial effects of adding spironolactone to recommended antihypertensive treatment in diabetic nephropathy: a randomized, double-masked, cross-over study. Diabetes Care. 2005 Sep;28(9):210612.

19 Sato A, Hayashi K, Naruse M, Saruta T. Effectiveness of aldosterone blockade in patients with diabetic nephropathy. Hypertension. 2003 Jan;41(1):64-8.

20 Bärfacker L, Kuhl A, Hillisch A, Grosser R, Figueroa-Pérez S, Heckroth H, et al. Discovery of BAY 94-8862: a nonsteroidal antagonist of the mineralocorticoid receptor for the treatment of cardiorenal diseases. ChemMedChem. 2012 Aug;7(8):1385-403.

21 Kolkhof P, Borden SA. Molecular pharmacology of the mineralocorticoid receptor: prospects for novel therapeutics. Mol Cell Endocrinol. 2012 Mar;350(2):310-7.

22 Kolkhof P, Delbeck M, Kretschmer A, Steinke W, Hartmann E, Bärfacker L, et al. Finerenone, a novel selective nonsteroidal mineralocorticoid receptor antagonist protects from rat cardiorenal injury. J Cardiovasc Pharmacol. 2014 Jul;64(1):69-78.

23 Grune J, Benz V, Brix S, Salatzki J, Blumrich A, Höft B, et al. Steroidal and nonsteroidal mineralocorticoid receptor antagonists cause differential cardiac gene expression in pressure overload-induced cardiac hypertrophy. J Cardiovasc Pharmacol. 2016 May;67(5):40211.

24 Grune J, Beyhoff N, Smeir E, Chudek R, Blumrich A, Ban Z, et al. Selective mineralocorticoid receptor cofactor modulation as molecular basis for finerenone's antifibrotic activity. Hypertension. 2018 Apr; 71(4): 599-608.

25 Jeunemaitre X, Chatellier G, Kreft-Jais C, Charru A, DeVries C, Plouin PF, et al. Efficacy and tolerance of spironolactone in essential hypertension. Am J Cardiol. 1987 Oct; 60(10):820-5.

26 Pitt B, Zannad F, Remme WJ, Cody R, Castaigne A, Perez A, et al.; Randomized Aldactone Evaluation Study Investigators. The effect of spironolactone on morbidity and mortality in patients with severe heart failure. N Engl J Med. 1999 Sep;341(10):70917.

27 Bakris GL, Agarwal R, Chan JC, Cooper ME, Gansevoort RT, Haller H, et al.; Mineralocor- ticoid Receptor Antagonist Tolerability Study-Diabetic Nephropathy (ARTS-DN) Study Group. Effect of finerenone on albuminuria in patients with diabetic nephropathy: a randomized clinical trial. JAMA. 2015 Sep;314(9):884-94.

28 Kidney Disease: Improving Global Outcomes (KDIGO) CKD Work Group. KDIGO 2012 clinical practice guideline for the evaluation and management of chronic kidney disease. 2013. [accessed July 30, 2019]. Available from: https://kdigo.org/wp-content/uploads/2017/02/KDIGO_2012_CKD_GL.pdf.

29 Zelniker TA, Wiviott SD, Raz I, Im K, Goodrich EL, Furtado RH, et al. Comparison of the effects of glucagon-like peptide receptor agonists and sodium-glucose cotransporter 2 inhibitors for prevention of major adverse cardiovascular and renal outcomes in type 2 diabetes mellitus. Circulation. 2019 Apr; 139(17):2022-31.

30 Fried LF, Emanuele N, Zhang JH, Brophy M, Conner TA, Duckworth W, et al.; VA NEPHRON-D Investigators. Combined angiotensin inhibition for the treatment of diabetic nephropathy. N Engl J Med. 2013 Nov;369(20): 1892-903.

31 Parving HH, Brenner BM, McMurray JJ, de Zeeuw D, Haffner SM, Solomon SD, et al.; ALTITUDE Investigators. Cardiorenal end points in a trial of aliskiren for type 2 diabetes. N Engl J Med. 2012 Dec;367(23):2204-13.

32 Zelniker TA, Wiviott SD, Raz I, Im K, Goodrich EL, Bonaca MP, et al. SGLT2 inhibitors for primary and secondary prevention of cardiovascular and renal outcomes in type 2 diabetes: a systematic review and meta-analysis of cardiovascular outcome trials. Lancet. 2019 Jan;393(10166):31-9.

33 Barrera-Chimal J, Girerd S, Jaisser F. Mineralocorticoid receptor antagonists and kidney diseases: pathophysiological basis. Kidney Int. 2019 Aug;96(2):302-19.

34 Coresh J, Heerspink HJ, Sang Y, Matsushita K, Arnlov J, Astor BC, et al.; Chronic Kidney Disease Prognosis Consortium and Chronic Kidney Disease Epidemiology Collaboration. Change in albuminuria and subsequent risk of end-stage kidney disease: an individual participant-level consortium meta-analysis of observational studies. Lancet Diabetes Endocrinol. 2019 Feb;7(2):115-27.

35 Dhaybi OA, Bakris G. Mineralocorticoid antagonists in chronic kidney disease. Curr Opin Nephrol Hypertens. 2017 Jan;26(1):505.

36 Yang CT, Kor CT, Hsieh YP. Long-term effects of spironolactone on kidney function and hyperkalemia-associated hospitalization in patients with chronic kidney disease. J Clin Med. 2018 Nov;7(11):459.

37 Trevisan M, de Deco P, Xu H, Evans M, Lindholm B, Bellocco R, et al. Incidence, predictors and clinical management of hyperkalaemia in new users of mineralocorticoid receptor antagonists. Eur J Heart Fail. 2018 Aug; 20(8):1217-26. 
38 Collins AJ, Pitt B, Reaven N, Funk S, McGaughey K, Wilson D, et al. Association of serum potassium with all-cause mortality in patients with and without heart failure, chronic kidney disease, and/or diabetes. Am J Nephrol. 2017;46(3):213-21.

39 Miao Y, Dobre D, Heerspink HJ, Brenner $\mathrm{BM}$, Cooper ME, Parving $\mathrm{HH}$, et al. Increased serum potassium affects renal outcomes: a post hoc analysis of the Reduction of Endpoints in NIDDM with the Angiotensin II Antagonist Losartan (RENAAL) trial. Diabetologia. 2011 Jan; 54(1):44-50.

40 Lazich I, Bakris GL. Prediction and management of hyperkalemia across the spectrum of chronic kidney disease. Semin Nephrol. 2014 May;34(3):333-9.
41 Pitt B, Kober L, Ponikowski P, Gheorghiade M, Filippatos G, Krum H, et al. Safety and tolerability of the novel non-steroidal mineralocorticoid receptor antagonist BAY 94-8862 in patients with chronic heart failure and mild or moderate chronic kidney disease: a randomized, double-blind trial. Eur Heart J. 2013 Aug;34(31):245363. 